\title{
A yeast platform for high-level synthesis of natural and unnatural tetrahydroisoquinoline
}

\section{alkaloids}

Michael E. Pyne ${ }^{\text {a,b }}$, Kaspar Kevvai ${ }^{\text {a,b,* }}$, Parbir S. Grewal ${ }^{\text {c,* }}$, Lauren Narcross ${ }^{\text {a,b }}$, Brian Choi $^{\text {, }}$ Leanne Bourgeois $^{\mathrm{a}, \mathrm{b}}$, John E. Dueber ${ }^{\mathrm{d}}$, \& Vincent J. J. Martin ${ }^{\mathrm{a}, \mathrm{b}}$,

a Department of Biology, Concordia University, Montréal, Québec, Canada

${ }^{\mathrm{b}}$ Centre for Applied Synthetic Biology, Concordia University, Montréal, Québec, Canada

${ }^{c}$ Department of Chemical and Biomolecular Engineering, University of California, Berkeley, Berkeley, California, USA

${ }^{\mathrm{d}}$ Department of Bioengineering, University of California, Berkeley, Berkeley, California, USA

* These authors contributed equally to this work

Correspondence and requests for materials should be addressed to: Vincent J.J. Martin (email: vincent.martin@ concordia.ca) 


\begin{abstract}
The tetrahydroisoquinoline (THIQ) moiety is a privileged substructure of many bioactive natural products and semi-synthetic analogues. The plant kingdom manufactures more than 3,000 THIQ alkaloids, including the opioids morphine and codeine. While microbial species have been engineered to synthesize a few compounds from the benzylisoquinoline alkaloid (BIA) family of THIQs, low product titers impede industrial viability and limit access to the full chemical space. Here we report a THIQ platform by increasing yeast production of the central BIA intermediate (S)-reticuline to more than $3 \mathrm{~g} \mathrm{~L}^{-1}$, a 38,000-fold improvement over our first-generation strain. Gains in BIA output coincided with the formation of several substituted THIQs derived from host amino acid catabolism. Enabled by this activity, we repurposed the yeast Ehrlich pathway and demonstrate the synthesis of an array of unnatural THIQ scaffolds. This work provides a blueprint for synthesizing new privileged structures and will enable the targeted overproduction of thousands of THIQ products, including natural and semi-synthetic opioids.
\end{abstract}

Keywords: Alkaloid, benzylisoquinoline, metabolic engineering, opioid, reticuline, tetrahydroisoquinoline 
Specialized metabolites equip plants with a chemical framework for communication and defense, whilst many such natural products have been exploited for use as flavors, dyes, and pharmaceuticals ${ }^{1}$. Crop-based manufacturing has enabled production of some plant metabolites at commercial scale ${ }^{2}$, yet low yields limit access to many high-value products. Although some natural products can be attained through chemical synthesis, the structural complexity of plant metabolites and a lack of stereocontrol result in unfeasible synthetic routes. Microbial biosynthesis has potential to overcome many of these hurdles and holds promise as a viable alternative to traditional modes of chemical and pharmaceutical manufacturing. Landmark successes in this arena, such as industrial-scale production of the anti-malarial precursor artemisinic acid $^{3}$ and the chemical building blocks 1,3-propanediol ${ }^{4}$ and $\beta$-farnesene ${ }^{5}$, have paved the way for new opportunities in microbial biomanufacturing.

The tetrahydroisoquinoline (THIQ) structural moiety forms the basis of more than 3,000 plant natural products ${ }^{6}$, as well as a suite of synthetic and semi-synthetic pharmaceuticals. Naturally-occurring THIQ metabolites include the benzylisoquinoline, phenethylisoquinoline, ipecac, and Amaryllidaceae alkaloid classes ${ }^{6}$. The peyote cactus produces a number of simple substituted and unsubstituted THIQs ${ }^{7}$, while species of Erythrina synthesize complex spiroTHIQ alkaloids ${ }^{8}$. Each of these metabolite classes possesses the privileged THIQ substructure that imparts a vast array of bioactivities following derivatization in downstream tailoring reactions. The Amaryllidaceae alkaloid galantamine and the phenethylisoquinoline colchicine are commercial THIQ-derived drugs used in the treatment of Alzheimer's disease and gout, respectively ${ }^{6}$. Synthetic and semi-synthetic THIQs approved by the FDA include the antiParkinson drug apomorphine, the chemotherapeutic trabectedin, the muscle relaxant 
cisatracurium, the anti-parasitic praziquantel, and the anti-chorea tetrabenazine used in the treatment of Huntington's disease ${ }^{9}$.

The benzylisoquinoline alkaloids (BIAs) are the largest class of THIQ natural products and include several of the most important human medicines ${ }^{10}$. Morphine, codeine, and their analogues are potent BIA analgesics included in the World Health Organization's List of Essential Medicines ${ }^{11}$. Papaverine is a vasodilator and antispasmodic drug, and noscapine exhibits promising anticancer properties ${ }^{10}$. Although global demand for morphinan BIAs is presently met through extraction from opium poppy (Papaver somniferum), most BIAs do not accumulate to sufficient concentrations in plant tissues. To begin exploring this untapped natural diversity, plant pathways mediating synthesis of noscapine, sanguinarine, morphine, codeine, and hydrocodone have been reconstructed in yeast ${ }^{12-15}$. Despite these achievements, present yeast BIA titers have been limited to less than $2 \mathrm{mg} \mathrm{L}^{-1}$ (ref. ${ }^{16-19}$ ). For instance, the hydrocodone pathway has been reconstituted within a single yeast strain at a titer of only $0.0003 \mathrm{mg} \mathrm{L}^{-1}$ (ref. ${ }^{17}$ ) largely due to inefficiencies in formation of the dedicated THIQ precursor. Higher levels of the key intermediate $(S)$-reticuline have been reached in $E$. coli cultures $\left(160 \mathrm{mg} \mathrm{L}^{-1}\right)^{20}$, yet bacteria lack membrane-bound organelles required for functional expression of the numerous cytochrome P450 enzymes in downstream BIA pathways. Consequently, engineering E. coli for total biosynthesis of morphinan BIAs required partitioning the pathway amongst four engineered strains $^{21}$. Scalable production of BIAs would be facilitated through the reconstruction of heterologous pathways in a single yeast strain.

An emerging objective of synthetic biology is directed at expanding natural product diversity by engineering structural scaffolds and chemical modifications that are not observed in nature $^{22}$. Although many biosynthetic enzymes exhibit broad substrate specificities when assayed 
in vitro, natural pathways have evolved a preference for a single substrate or very small subset of accepted building blocks ${ }^{23}$. The Pictet-Spengler condensation between an aryl amine and a carbonyl compound underlies the synthesis of more than 3,000 THIQ alkaloids ${ }^{6}$, yet much of this diversity arises from only four aldehyde species (4-hydroxyphenylacetaldehyde, 4hydroxydihydrocinnamaldehyde, protocatechuic aldehyde, and secologanin). Because PictetSpenglerases are able to accept a tremendous range of carbonyl substrates ${ }^{8,24-27}$, natural THIQ alkaloids occupy a miniscule fraction of the conceivable chemical space. To access this untapped potential, norcoclaurine synthase (NCS) has been exploited for the synthesis of novel substituted THIQs ${ }^{25,27}$, including ones derived from ketone building blocks ${ }^{8}$. These in vitro approaches involve the synthesis of complex and unusual carbonyl substrates; consequently, producing such compounds at scale presents a formidable challenge.

We set out to establish a high-level biosynthetic route to THIQ alkaloids in which diverse structures are synthesized in vivo from simple substrates. We increased yeast output of the committed BIA intermediate $(S)$-reticuline to $3.1 \mathrm{~g} \mathrm{~L}^{-1}$, exemplifying a more than 38,000 -fold improvement over our previous work ${ }^{16}$. These improvements shorten the path to industrial-scale production of existing BIA pharmaceuticals and will enable the targeted overproduction of thousands of natural structures that remain uncharacterized from a pharmacological perspective. We further exploited the biosynthetic potential of our THIQ platform by synthesizing a suite of unnatural THIQ scaffolds from both endogenous and supplemented amino acids. These efforts expand the diversity of privileged THIQ structures beyond natural products and outline a general synthetic biology framework for building new chemical scaffolds in yeast. 


\section{RESULTS}

\section{A yeast platform for high-level synthesis of substituted tetrahydroisoquinolines}

To improve biosynthesis of THIQs in yeast, we focused on the first committed reaction and major rate-limiting step of the canonical BIA pathway. This conversion involves condensation of 4-hydroxyphenylacetaldehyde (4-HPAA, 1) and dopamine (2) by NCS, yielding $(S)$ -

norcoclaurine (3) (Fig. 1a). We improved production of (S)-norcoclaurine more than 11,000-fold compared to our previous efforts ${ }^{16}$ by implementing $>20$ successive strain modifications to the yeast shikimate, Ehrlich, and L-tyrosine metabolic pathways (Fig. 1b; strain LP478). (S)Norcoclaurine synthesis in our early strains was limited by 4-HPAA, which is rapidly transformed to the corresponding fusel acid (4-hydroxyphenylacetate; 4-HPAC, 4) or alcohol $($ tyrosol, 5) via the Ehrlich pathway. The specific enzymes mediating these redox reactions have thus far evaded identification ${ }^{28}$, as yeast produces a suite of more than 30 oxidoreductases $^{29}$. A gene deletion screen of more than 20 of these candidates implicated the Ari1 short-chain dehydrogenase/reductase in 4-HPAA reduction (Supplementary Fig. 1). Owing to redundancy in yeast oxidoreductases, we proceeded to delete a total of five genes encoding NADPHdependent reductases and dehydrogenases (in order: $\operatorname{aril} \Delta \operatorname{adh} 6 \Delta \operatorname{ypr} 1 \Delta y \operatorname{dr} 541 c \Delta \operatorname{aad} 3 \Delta$ ), enabling the production of $77 \mathrm{mg} \mathrm{L}^{-1}$ of $(S)$-norcoclaurine by strain LP386 (Fig. 1b and Supplementary Fig. 2). Although the ALD4 aldehyde-dehydrogenase-encoding gene was deleted in early strains, resulting in gains in both dopamine and $(S)$-norcoclaurine titer (Supplementary Fig. 1 and 2), we reintroduced the gene in later strains to promote ethanol consumption in fed-batch fermentor cultures (Fig. 1b; strains LP474 and LP478). To further improve precursor supply, strain LP478 incorporates overexpression of genes encoding chorismate synthase (ARO2), prephenate dehydrogenase (TYRI), and phenylpyruvate 
decarboxylase (ARO10), as well as feedback-resistant forms of 3-deoxy-D-arabino-

heptulosonate-7-phosphate synthase $\left(A R O 4^{F B R}\right)$ and chorismate mutase $\left(A R O 7^{F B R}\right)$. We also

inactivated the L-phenylalanine and L-tryptophan biosynthetic pathways through deletion of

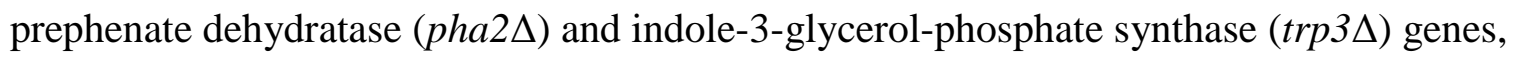

respectively.

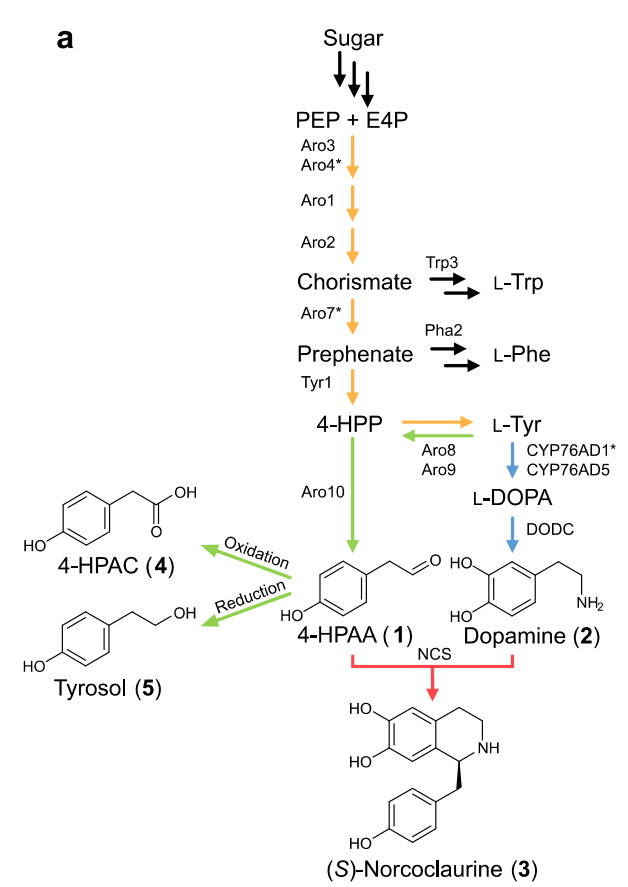

b

Fig. 1 - Engineering a THIQ-producing yeast. a, $(S)$-Norcoclaurine $(3)$ synthetic pathway in engineered yeast. The native yeast Ehrlich pathway (green) supplies 4-HPAA (1) from L-tyrosine, while a heterologous pathway (blue) generates dopamine (2), also from L-tyrosine. CYP76AD5 is a more active plant tyrosine hydroxylase compared to CYP76AD1* (CYP76AD1 ${ }^{\mathrm{W} 13 \mathrm{~L}}{ }^{\mathrm{F} 309 \mathrm{~L}}$ ), which is an engineered variant of CYP76AD1. NCS catalyzes an enantioselective Pictet-Spengler condensation of 4-HPAA and dopamine, yielding $(S)$-norcoclaurine (pink). Native shikimate and L-tyrosine biosynthetic pathways are shown in orange. b, $(S)$-Norcoclaurine (3) titer in culture supernatants of successive engineered strains. Error bars represent s.d. of three biological replicates. All strains exhibited a significant increase $(P<0.05)$ in $(S)$-norcoclaurine titer relative to the respective parental strain, with the exception of strain LP442, which exhibited a significant decrease $(P<0.05)$ in titer relative to strain LP412. Refer to Supplementary Table 4 for genotypes of the full 23-strain (S)-norcoclaurine lineage. Abbreviations: L-DOPA, L-3,4dihydroxyphenylalanine; DODC, DOPA decarboxylase; E4P, erythrose-4-phosphate; 4-HPAA, 4hydroxyphenylacetaldehyde; 4-HPAC, 4-hydroxyphenylacetate; NCS, norcoclaurine synthase; PEP, phosphoenolpyruvate; L-Phe, L-phenylalanine; L-Trp, L-tryptophan; L-Tyr, L-tyrosine.

Our initial $(S)$-norcoclaurine-producing strains contained an N-terminally truncated NCS variant from Nandina domestica $\left(N d \mathrm{NCS} \Delta \mathrm{N}_{20}\right)^{30}$ (Supplementary Fig. 3). Because $(S)$ norcoclaurine production increased by supplying additional copies of the $N d N C S \triangle N_{20}$ gene (up 
to eight copies) (Fig. 1b and Supplementary Fig. 2), we set out to identify a more efficient NCS ortholog. Several groups have reported the use of truncation variants of NCS from Coptis japonica $(C j \mathrm{NCS})^{18,25}$. We explored several extensive N-terminal truncations of $C j \mathrm{NCS}$ by reducing the protein to the core Bet v1 domain and comparing NCS activity to $N d \mathrm{NCS} \Delta \mathrm{N}_{20}$. A 35 amino acid deletion ( $\left.C j \mathrm{NCS} \Delta \mathrm{N}_{35}\right)$ facilitated the production of five-fold more $(S)$ norcoclaurine than an isogenic strain harboring $N d \mathrm{NCS} \Delta \mathrm{N}_{20}$ (Supplementary Fig. 4). Implementation of $C j \mathrm{NCS} \Delta \mathrm{N}_{35}$ in strain LP386 possessing eight copies of $N d N C S \triangle N_{20}$ facilitated a 50\% increase in (S)-norcoclaurine titer (strain LP412). We proceeded to delete all eight copies of $N d N C S \triangle N_{20}$ (strain LP442) and integrated an additional copy of CjNCS $\triangle N_{35}$ (strain LP474).

Concurrent precursor feeding experiments revealed a greater increase in $(S)$ norcoclaurine production upon supplementation of L-DOPA compared to L-tyrosine (Supplementary Fig. 5), indicating a limitation in dopamine supply. To address this substrate imbalance, we introduced a superior tyrosine hydroxylase ortholog (CYP76AD5) ${ }^{31-34}$ into strain LP474, which already contains an engineered CYP76AD1 variant (CYP76AD $\left.1^{\text {W13L F309L }}\right)^{16}$ (Supplementary Fig. 6; strain LP478). In line with substrate supplementation experiments, increased expression of tyrosine hydroxylase through implementation of $C Y P 76 A D 5$ doubled $(S)$-norcoclaurine titer $\left(245 \mathrm{mg} \mathrm{L}^{-1}\right)$ relative to strain LP474. Cultivation of strain LP478 in a pulsed sucrose fed-batch fermentor yielded $1.6 \mathrm{~g} \mathrm{~L}^{-1}$ of $(S)$-norcoclaurine and $2.1 \mathrm{~g} \mathrm{~L}^{-1}$ of tyrosol (Supplementary Fig. 7). Thus, applying a fed-batch process to our engineered S. cerevisiae strain enabled unprecedented titers of BIAs that are synthesized directly from sugar. 


\section{Production of functionalized substituted THIQs}

Having achieved $>1 \mathrm{~g} \mathrm{~L}^{-1}$ of $(S)$-norcoclaurine, we sought to extend the BIA pathway to the major branch point intermediate $(S)$-reticuline. This four-step conversion involves methylation of the THIQ motif of $(S)$-norcoclaurine (3) by two methyltransferases from opium poppy (Papaver somniferum; Ps6OMT and PsCNMT), yielding $(S)$-coclaurine (8) followed by $(S)-N$ methylcoclaurine (9) (Fig. 2a). The benzyl moiety of (S)-N-methylcoclaurine is then hydroxylated by a cytochrome P450 N-methylcoclaurine hydroxylase from California poppy (Eschscholzia californica; CYP80B1 or EcNMCH) ${ }^{16}$, yielding $(S)-3^{\prime}$-hydroxy- $N$ methylcoclaurine (10), which is subsequently methylated by a $4^{\prime} O$-methyltransferase (Ps4'OMT2) to generate (S)-reticuline (11). Our (S)-reticuline pathway module included a cytochrome P450 reductase (CPR) from Arabidopsis thaliana (AtATR2) for electron transfer to $E_{c} \mathrm{NMCH}$. Introduction of this composite pathway to strain LP478 generated $270 \mathrm{mg} \mathrm{L}^{-1}$ of $(S)$ reticuline in microtiter plate cultivations (Fig. 2b; strain LP490). All three intermediates in the conversion of $(S)$-norcoclaurine (3) to $(S)$-reticuline (11) were detected. Assuming an equal ionization efficiency, $(S)$-3'-hydroxy- $N$-methylcoclaurine (10) was found to accumulate to the greatest extent. To address this bottleneck, we integrated an additional copy of Ps4'OMT2 (strain LP491), which nearly abolished accumulation of (S)-3'-hydroxy- $N$-methylcoclaurine and resulted in a $76 \%$ improvement in $(S)$-reticuline titer to $476 \mathrm{mg} \mathrm{L}^{-1}$.

We measured fusel products generated by strain LP491 in microtiter plate cultures and observed substantial accumulation of tyrosol $\left(235 \mathrm{mg} \mathrm{L}^{-1}\right)$ despite deletion of five oxidoreductase genes, implying that additional unidentified enzymes reduce 4-HPAA to tyrosol. Single-gene deletions of seven of these candidates (AAD4, AAD14, ADH7, GCY1, GRE2, SFA1, and YGLO39W) in strain LP491 identified Gre2 as a potent 4-HPAA reductase (Supplementary Fig. 
8), as its inactivation facilitated an $83 \%$ decrease in tyrosol formation in microtiter plate cultures

(Supplementary Fig. 9; strain LP494). Although (S)-reticuline titer was unaltered under these

conditions, $(S)$-norcoclaurine production more than doubled $\left(111 \mathrm{mg} \mathrm{L}^{-1}\right.$ to $\left.263 \mathrm{mg} \mathrm{L}^{-1}\right)$ (Fig.

2b). Growth of strain LP494 in a pulsed fed-batch fermentor produced only $138 \mathrm{mg} \mathrm{L}^{-1}$ of

tyrosol, whereas the $(S)$-norcoclaurine-producing strain LP478 cultivated under identical

conditions generated $2.1 \mathrm{~g} \mathrm{~L}^{-1}$ of tyrosol (Supplementary Fig. 7), corresponding to a 93\%

reduction in tyrosol synthesis with improved flux to the heterologous pathway (Supplementary

Fig. 9).

a<smiles>Oc1ccc(CC2NCCc3cc(O)c(O)cc32)cc1</smiles>

(S)-Norcoclaurine (3)<smiles>COc1cc2c(cc1O)[C@@H](Cc1ccc(O)cc1)NCC2</smiles>

(S)-Coclaurine (8)

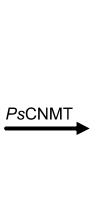

(9)

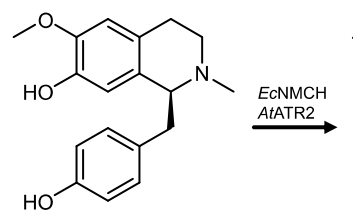

(9)<smiles>COc1cc2c(cc1O)C(Cc1ccc(O)c(O)c1)N(C)CC2</smiles>

(S)-3'-Hydroxy-NMethylcoclaurine (10)<smiles>COc1ccc(CC2c3cc(O)c(OC)cc3CCN2C)cc1O</smiles>

(S)-Reticuline (11)
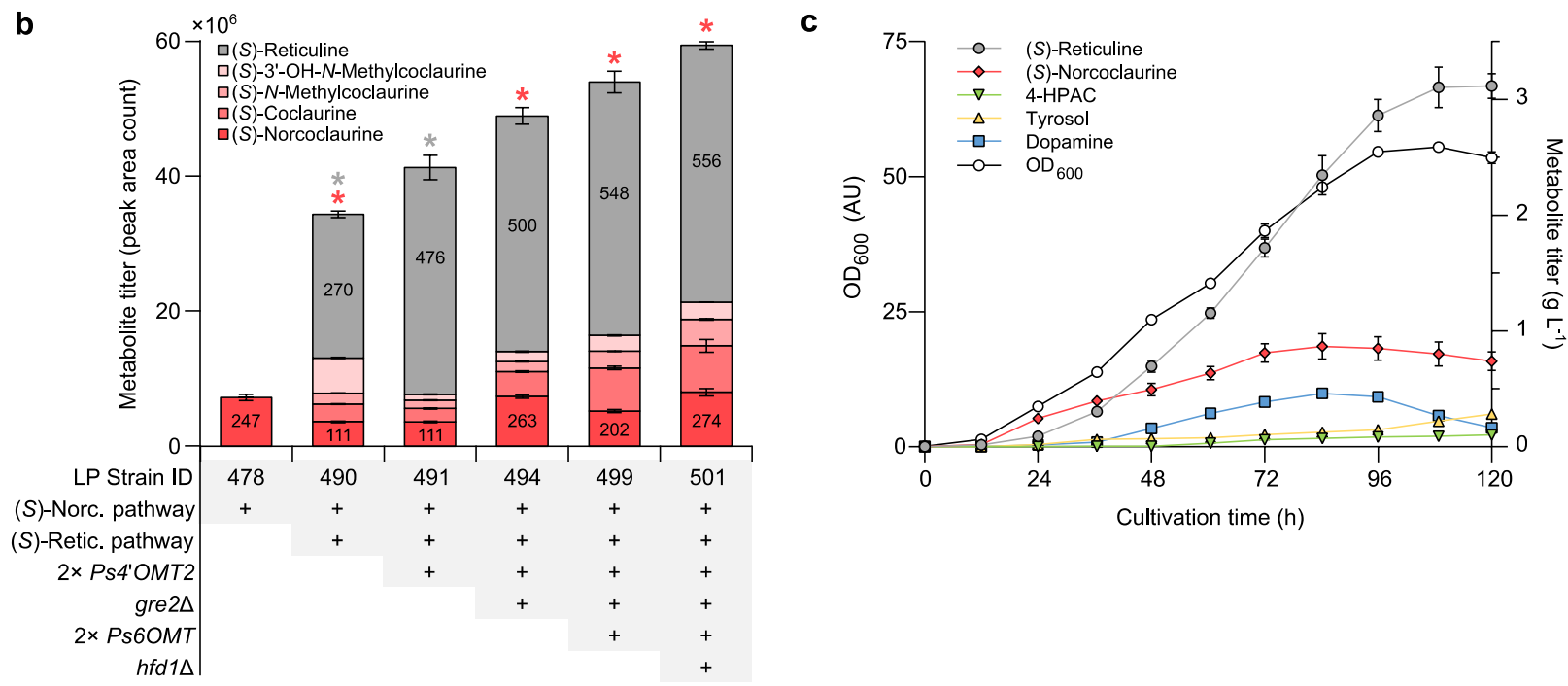

Fig. 2 -Extending the canonical THIQ pathway to $(S)$-reticuline. a, Composite pathway for synthesis of $(S)$ reticuline (11) from $(S)$-norcoclaurine $(\mathbf{3})$. b, Relative titers of $(S)$-reticuline pathway intermediates in culture supernatants of successive engineered production strains. Peak area counts are shown for all pathway intermediates and absolute titers are depicted in $\mathrm{mg} \mathrm{L}^{-1}$ for $(S)$-norcoclaurine and $(S)$-reticuline. Error bars represent s.d. of three biological replicates. Asterisk $(*)$ denotes a significant increase or decrease $(P<0.05)$ in $(S)$-norcoclaurine or $(S)$ reticuline titer relative to the parental strain. c, Cultivation of an $(S)$-reticuline-producing strain (LP501 harboring pHUM) in a sucrose-pulsed fed-batch fermentor. Growth of biomass $\left(\mathrm{OD}_{600}\right)$ and accumulation of BIA metabolites in the culture medium during cultivation. Error bars represent s.e.m. of duplicate experiments. 
With tyrosol production nearly abolished, 4-HPAC increased in concentration and became the dominant fusel product in both microtiter plate and pulsed fed-batch fermentor cultures (Supplementary Fig. 9). We thus set out to identify the enzyme(s) responsible for the oxidation of 4-HPAA to 4-HPAC. We surveyed yeast aldehyde dehydrogenases for cytosolic enzymes that exhibit activity on aromatic aldehydes. Although Ald2 and Ald3 meet these criteria, deletion of both $A L D 2$ and ALD3 in strain LP492 (resulting in strain LP495) failed to alter 4-HPAC concentration in microtiter plate cultures (data not shown). We then turned our attention to Hfd1, a dual-function aldehyde dehydrogenase involved in ubiquinone (coenzyme $\mathrm{Q}_{10}$ ) biosynthesis and fatty acid catabolism. One of the physiological substrates of Hfd1 is 4hydroxybenzaldehyde ${ }^{35}$, a close structural analogue of 4-HPAA, and localization studies have shown that Hfd1 resides in the outer mitochondrial membrane where it is ostensibly exposed to the cytosol ${ }^{36}$. Deletion of HFD1 in our LP494 gre $2 \Delta$ mutant strain (yielding strain LP498) decreased 4-HPAC production by more than $80 \%$ in both microtiter plate and pulsed fed-batch fermentor cultures (Supplementary Fig. 10). Our final $(S)$-reticuline production strain (LP501) incorporates gre $2 \Delta$ and $h f d 1 \Delta$ mutations for reduced synthesis of fusel products, as well as an additional copy of Ps6OMT to improve conversion of $(S)$-norcoclaurine to $(S)$-coclaurine. Strain LP501 produced $3.1 \mathrm{~g} \mathrm{~L}^{-1}$ of $(S)$-reticuline and $0.7 \mathrm{~g} \mathrm{~L}^{-1}$ of $(S)$-norcoclaurine in a pulsed fedbatch fermentor (Fig. 2c). Tyrosol, 4-HPAC, and dopamine reached final concentrations of less than $0.3 \mathrm{~g} \mathrm{~L}^{-1}$.

\section{De novo synthesis of unnatural substituted tetrahydroisoquinolines}

In addition to benzylisoquinolines, higher plants synthesize phenethylisoquinolines and the Amaryllidaceae alkaloids via 4-hydroxydihydrocinnamaldehyde and protocatechuic aldehyde, 
respectively. These natural pathways as well as in vitro studies $^{8,25,27,37}$ highlight the broad substrate specificity of Pictet-Spenglerases and prompted us to employ our engineered strains to explore NCS promiscuity. We examined LC-MS spectra derived from supernatants of an $(S)$ norcoclaurine production strain for peaks indicative of substituted THIQ products. Our search consisted of 64 theoretical THIQ products derived through Pictet-Spengler condensation of dopamine and endogenous yeast carbonyl species. From this search we identified four putative LC-MS peaks (Supplementary Table 1). A major peak corresponding to salsolinol (13), derived from condensation of dopamine (2) and acetaldehyde (12) (Fig. 3a), was observed in supernatants of all dopamine-producing strains irrespective of the presence of an NCS biosynthetic enzyme. Three additional LC-MS peaks were consistent with substituted THIQs derived from condensation of dopamine (2) and aldehydes from the Ehrlich pathway ${ }^{28}$. These substituted THIQs (16, 19, and 22) arise from L-phenylalanine (14), L-tryptophan (17), and Lleucine (20) catabolism via the respective aldehydes phenylacetaldehyde (PAA, 15), indole acetaldehyde (IAA, 18), and 3-methylbutanal (3MB, 21) (Fig. 3b). Targeted fragmentation of substituted THIQ products yielded spectra consistent with the presumed metabolite identities ${ }^{38}$ (Supplementary Fig. 11). Further, cultivating strain LP385 harboring $C j \mathrm{NCS} \Delta \mathrm{N}_{35}$ on L-tyrosine (6), L-phenylalanine (14), L-tryptophan (17), or L-leucine (20) as a sole nitrogen source increased the area of LC-MS peaks corresponding to $(S)$-norcoclaurine $(3)$ and products 16, 19, and 22, respectively (Supplementary Fig. 12), providing additional evidence that these substituted THIQs arise from amino acid degradation. Analogous substituted THIQs derived from catabolism of L-isoleucine and L-valine were not observed since $\alpha$-substituted aldehydes are not well tolerated by $\mathrm{NCS}^{39}$. 
a

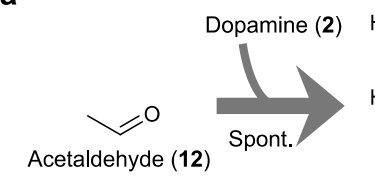

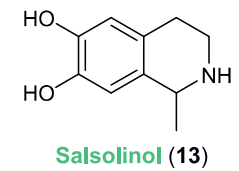

b

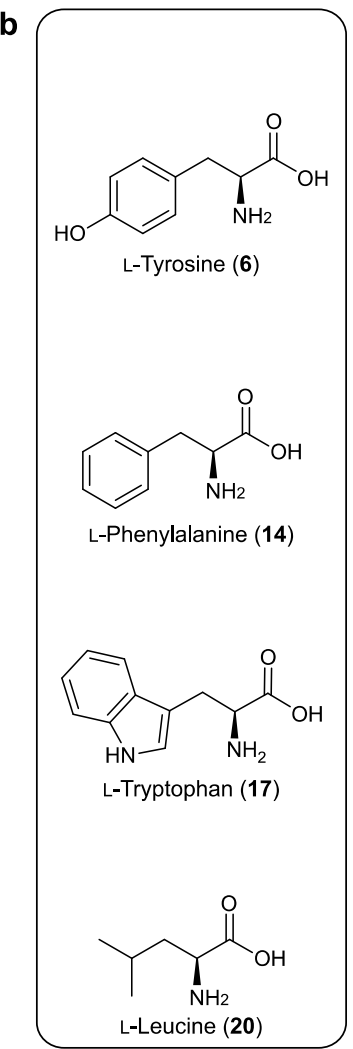

Salsolinol (13)

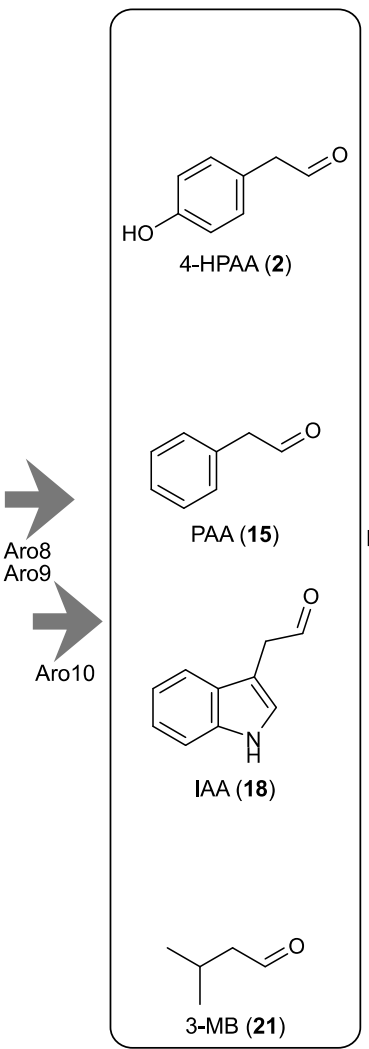

C
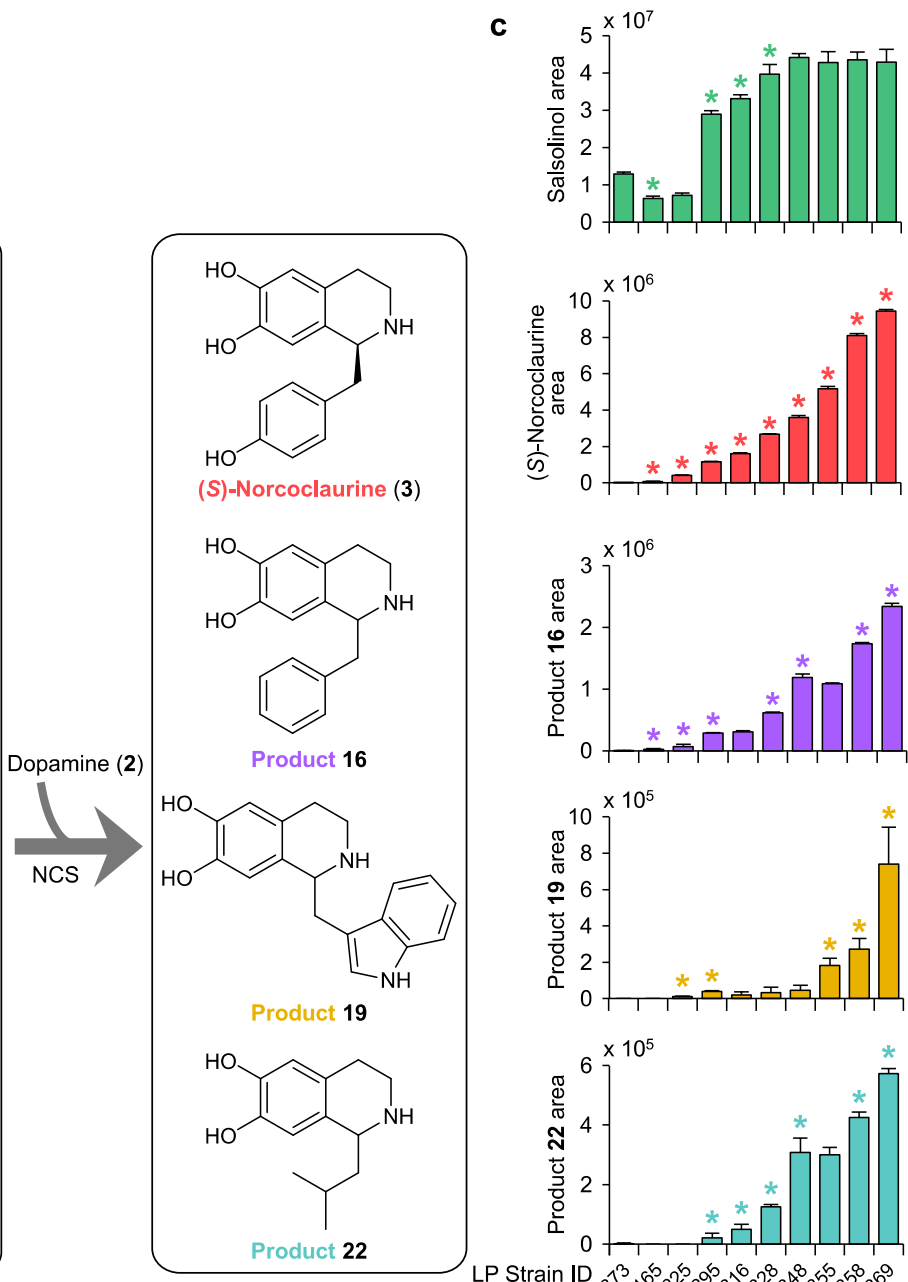
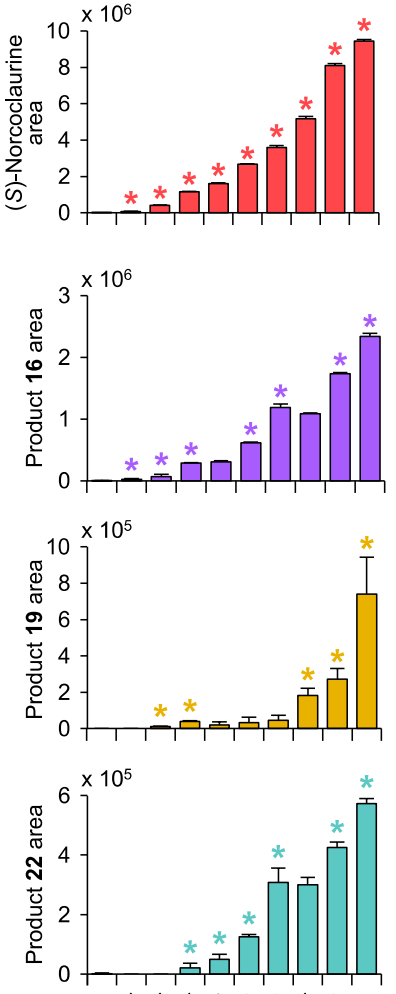

Fig. 3 - De novo synthesis of substituted tetrahydroisoquinolines in strains engineered for $(S)$-norcoclaurine production. a, Formation of salsolinol (13) from acetaldehyde (12) and dopamine (2) occurs spontaneously in yeast strains engineered for dopamine production. b, NCS-catalyzed formation of substituted THIQs through catabolism of endogenous amino acids via the Ehrlich pathway. Amino acids are converted to the respective aldehyde species via sequential transamination (Aro8/Aro9) and decarboxylation (Aro10) reactions. In the presence of dopamine (2) and NCS, aldehydes are converted to the corresponding substituted THIQs. Stereochemistry of unnatural substituted THIQs is omitted. c, Relative levels of substituted THIQs in yeast strains engineered for $(S)$-norcoclaurine (3) production. Strain 1373 possesses the dopamine pathway and lacks an NCS biosynthetic enzyme. Refer to Fig. $1 \mathrm{~b}$ and Supplementary Table 4 for genotypes of engineered strains. Error bars represent s.d. of three biological replicates. Asterisk $(*)$ denotes a significant increase or decrease $(P<0.05)$ in product titer relative to the precursor strain. Abbreviations: 4-HPAA, 4-hydroxyphenylacetaldehyde; IAA, indole acetaldehyde; 3-MB, 3-methylbutanal; PAA, phenylacetaldehyde; spont., spontaneous.

\section{We analyzed LC-MS spectra from supernatants of our oxidoreductase gene deletion}

strains to monitor levels of substituted THIQ products $(\mathbf{1 6}, \mathbf{1 9}$, and 22) relative to improvements

in $(S)$-norcoclaurine concentration (Fig. 3c). These THIQs were not identified in supernatants of

a dopamine-producing strain lacking an NCS enzyme (strain 1373) and, with the exception of 16, 
were not detected in the $N d \mathrm{NCS}$ strain used as the basis of our oxidoreductase gene deletions (strain LP165). Overall, formation of these products closely paralleled $(S)$-norcoclaurine, in which biosynthesis of 16, 19, and 22 increased in accordance with $(S)$-norcoclaurine throughout the oxidoreductase gene deletion lineage. Synthesis of $\mathbf{1 6}$ and $\mathbf{1 9}$ increased through deletion of $A R I 1$, whereas deletion of $Y P R I$ or $A D H 6$ increased production of 19 and 22, respectively. Deletion of YDR541C enhanced levels of all THIQs derived from the Ehrlich pathway.

\section{Synthesis of unnatural substituted tetrahydroisoquinolines from supplemented amino acids}

Owing to the capacity of our engineered host to synthesize substituted THIQs from endogenous amino acids, we reasoned that supplying exogenous amino acids would enable the synthesis of additional THIQ structures. We devised a THIQ synthesis assay by cultivating our $(S)$-reticuline production strain (LP501) on individual amino acids as the major source of nitrogen. In this manner, amino acid utilization via the Ehrlich pathway directly links cell growth to aldehyde and thus THIQ formation. Using this assay, we synthesized a diverse set of THIQs possessing both aliphatic and aromatic substitutions (Supplementary Fig. 13 and Supplementary Table 2). Supplying L-methionine (23) as the major nitrogen source generated the sulfur-containing THIQ 25 via methional (24) (Fig. 4). L-DOPA is a non-proteinogenic derivative of L-tyrosine, suggesting that it serves as a substrate for enzymes of the Ehrlich pathway. Feeding L-DOPA (7) to strain LP501 gave rise to norlaudanosoline (tetrahydropapaveroline, 28) via 3,4dihydroxyphenylacetaldehyde (3,4-dHPAA, 27). We also demonstrated production of ethyl-, propyl-, butyl-, and pentyl-substituted THIQs $(\mathbf{3 1}, \mathbf{3 5}, \mathbf{3 9}$, and 43, respectively) by supplying L2-aminobutyrate (29), L-norvaline (33), L-norleucine (37), and L-2-aminoheptanoic acid (41), 
respectively (Fig. 4). Targeted fragmentation of substituted THIQs yielded spectra consistent with the presumed metabolite identities ${ }^{38}$ (Supplementary Fig. 14).

a

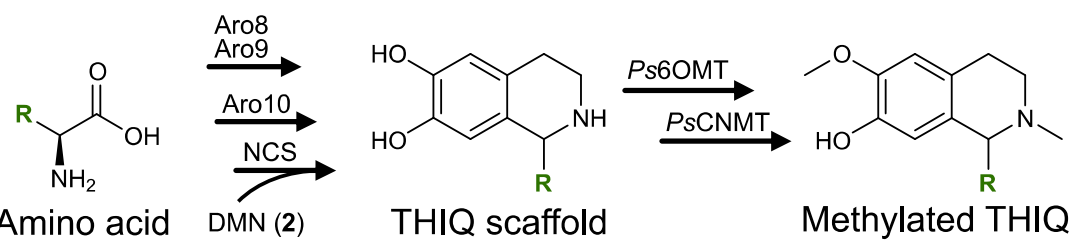

b<smiles>CC(C)Cc1ccc(O[Al-])cc1</smiles><smiles>CSCCC(C)C</smiles>
L-Methionine (23)<smiles>CC(C)(C)Cc1cc(O)c(O)c([18OH])c1</smiles><smiles>C1CCCCC1</smiles>

L-2-Aminobutyrate Tam L-Norvaline (33) L-2-Aminoheptanoic acid (41) (41)
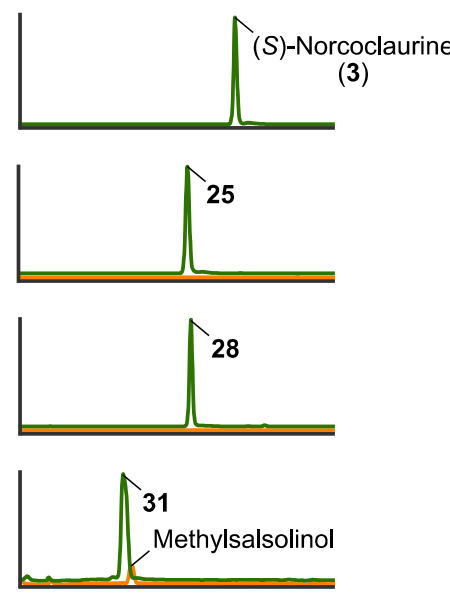

(29)
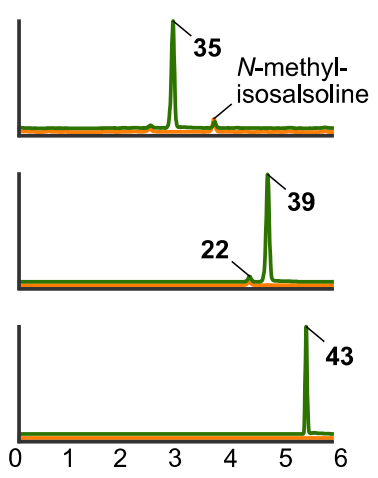
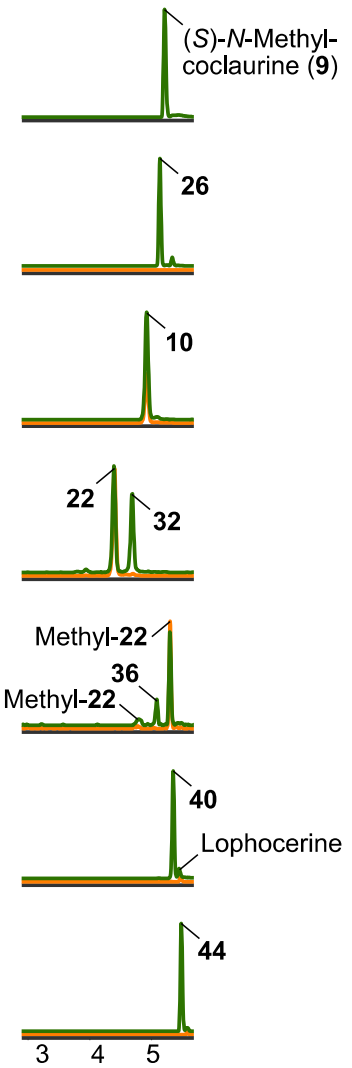

Fig. 4 - Synthesis and modification of unnatural substituted tetrahydroisoquinolines from supplemented amino acids. a, NCS-catalyzed formation of substituted THIQs through catabolism of externally supplied amino acids via the Ehrlich pathway. b, Ion-extracted LC-MS chromatograms of strain LP501 grown on L-methionine (23), L-DOPA (7), L-2-aminobutyrate (29), L-norvaline (33), L-norleucine (37), or L-2-aminoheptanoic acid (41) as the chief source of nitrogen (green). Aldehydes derived from amino acids were incorporated into the corresponding substituted THIQs and methylated by BIA tailoring enzymes (Ps6OMT and PsCNMT) produced by strain LP501. Substituted THIQs and their methylated derivatives shifted in retention time relative to the canonical BIA products from L-tyrosine, namely $(S)$-norcoclaurine (3) and $(S)$ - $N$-methylcoclaurine (9), which were formed de novo on all amino acid substrates. Growth of strain LP501 on urea as the major nitrogen source (orange) failed to generate peaks corresponding to substituted THIQs with the exception of $\mathbf{3 1}$ and $\mathbf{4 0}$, which co-elute with singly-methylated salsolinol (by Ps6OMT or $P s C N M T$ ) and lophocerine, respectively. Product $\mathbf{3 6}$ elutes closely with both singly-methylated derivatives of 22 (by $P s 6 \mathrm{OMT}$ or PsCNMT), which are synthesized de novo from L-leucine. Products $\mathbf{3 5}$ and $\mathbf{3 9}$ are isomers of $N$ methylisosalsoline and 22, respectively, but do not co-elute with these de novo products. Methylation of norlaudanosoline (28) by Ps6OMT and PsCNMT yields 3-hydroxy- $N$-methylcoclaurine (10), which is produced $d e$ novo by strain LP501 irrespective of the nitrogen source. All $\mathrm{m} / \mathrm{z}$ values were calculated based on the expected structures of the respective compounds of interest (Supplementary Fig. 13 and Supplementary Table 2). Abbreviations: DMN, dopamine. 
We also explored the promiscuity of BIA tailoring enzymes toward unnatural substituted THIQs. Enzymes involved in methylation of $(S)$-norcoclaurine (Ps6OMT and PsCNMT) exhibited activity on all substituted THIQs derived from supplemented amino acids (Fig. 4), as well as THIQ scaffolds synthesized de novo from endogenous substrates (Supplementary Fig. 15). Methylation of salsolinol (13) by Ps6OMT and PsCNMT yielded $N$-methylisosalsoline (45), while methylation of the THIQ scaffold derived from L-leucine (22) gave rise to lophocerine (48), a naturally occurring THIQ alkaloid ${ }^{40}$. Thus, the pool of BIAs and other THIQs that can be synthesized using our high-flux THIQ-producing yeast is immensely diverse.

\section{DISCUSSION}

Our work demonstrates a major step towards industrial synthesis of microbially-sourced THIQ pharmaceuticals by increasing production of the key BIA intermediate $(S)$-reticuline to $3.1 \mathrm{~g} \mathrm{~L}^{-1}$. Many bioprocesses become commercially viable when titers reach the gram per liter level and a target of $5 \mathrm{~g} \mathrm{~L}^{-1}$ has been set forth for commercial-scale production of opioids ${ }^{17}$. Although our titers are commensurate with this benchmark, morphine is synthesized several enzymatic steps downstream of $(S)$-reticuline. The efficiency of these reactions within a high titer production host remains unknown, yet the recent discovery of dedicated thebaine and neopinone biosynthetic enzymes $^{41,42}$ primes our engineered yeast for production of opioid pharmaceuticals at scale. Beyond natural products, our microbial production platform also enables the production of diverse molecules not sampled in nature.

Owing to their reactivity and toxicity within biological systems, microorganisms rapidly transform aldehydes to acids or alcohols ${ }^{43}$. Our initial BIA-producing strains were found to be limited by 4-HPAA, which is converted to tyrosol or 4-HPAC by a suite of hitherto unidentified 
enzymes $^{16,44}$. We have resolved this long-standing issue by identifying six redundant

oxidoreductases involved in tyrosol formation (Ari1, Adh6, Ypr1, Ydr541c, Aad3, and Gre2), in addition to the major 4-HPAC-forming enzyme (Hfd1). These oxidoreductases are involved in the catabolism of multiple Ehrlich pathway amino acids, as their inactivation triggered the biosynthesis of THIQs derived from L-phenylalanine, L-tryptophan, and L-leucine. With respect to fusel alcohol synthesis, most prior reports have implicated the ADH family of largely NADHdependent dehydrogenases ${ }^{28,45}$, whilst we have shown that exclusively NADPH-dependent enzymes mediate tyrosol formation. Relatedly, identification of Hfd1 as the chief 4-HPACforming enzyme contrasts the widely held notion that exclusively ALD-family enzymes catalyze the oxidation of fusel aldehydes in yeast ${ }^{28}$. These findings have implications within the food and beverage industries, in which production of fusel products is a key determinant of flavor profiles $^{28}$. More broadly, the capacity of our engineered strain to synthesize high levels of both aliphatic and aromatic aldehydes suggests that it could be repurposed to produce diverse compounds derived from carbonyl intermediates, for instance biofuels, fragrances, and chemotherapeutics $^{28,46-48}$.

Our THIQ production host enables the synthesis of new privileged structures by diverting carbonyl intermediates of the yeast Ehrlich pathway to THIQ synthesis. We exploited this activity to synthesize several unnatural THIQ analogues by supplying a suite of aromatic and aliphatic building blocks to our engineered host. To our knowledge most of these structures have not been observed in nature and thus our work broadens the chemical space occupied by natural THIQ products. It is noteworthy that the isobutyl-substituted THIQ (22) derived from L-leucine has been identified in the senita cactus (Lophocereus schotti) where it forms the basis of the methylated alkaloid lophocerine ${ }^{40}$. Our $(S)$-reticuline production host recapitulated this natural 
pathway, as implementation of Ps6OMT and PsCNMT resulted in de novo synthesis of lophocerine. We also observed the formation of salsolinol and its methylated derivatives, demonstrating that diverse THIQ scaffolds can be furnished with functional groups using canonical BIA tailoring enzymes. Furthermore, the THIQ scaffold formed from PAA (16) possesses the core BIA skeleton, indicating that the benzyl moiety of BIAs can also derive from L-phenylalanine. Products arising from 16 would lack hydroxylation at the $4^{\prime}$ position of the benzyl substituent that originates from 4-HPAA and is present in nearly all BIA structures characterized to date. In this context, sacred lotus (Nelumbo nucifera) synthesizes aporphine alkaloids possessing an unsubstituted benzyl moiety ${ }^{49}$ and thus our work exposes a plausible biosynthetic route to these distinct BIAs.

In summary, we engineered a yeast host capable of synthesizing high titers of the central BIA intermediate $(S)$-reticuline. This metabolite is a direct precursor to all natural BIAs, including the morphinan family, providing a platform for the microbial synthesis of natural opiates and their semi-synthetic derivatives. Microbial biosynthesis also provides the opportunity to access thousands of untapped metabolites that are present at low levels in source plants, a goal that is now within reach given our improvements in yeast THIQ biosynthesis. Finally, linking plant BIA metabolism to our repurposed Ehrlich pathway afforded yeast with the capacity to synthesize diverse new THIQ scaffolds. The THIQ motif is a privileged substructure that primes many natural products for bioactivity and thus harnessing this newfound activity expands the diversity of THIQ alkaloids beyond the canonical L-tyrosine pathway and will bring about the development of alkaloids with new pharmacological properties. 


\section{Acknowledgments}

We thank Marcos DiFalco for assistance with LC-MS analyses, as well as Chris Law and the Centre for Microscopy and Cellular Imaging, which is funded by Concordia University and the Canada Foundation for Innovation. This study was financially supported by an NSERCIndustrial Biocatalysis Network (IBN) grant, an NSERC Discovery grant, and by River Stone Biotech ApS. M.E.P. was supported by an NSERC Postdoctoral Fellowship, K.K. was supported by a Concordia University Horizon Postdoctoral Fellowship, L.N. was supported by a FQRNT DE Doctoral Research Scholarship for Foreign Students, L.B. was supported by an NSER Concordia graduate scholarship. J.E.D. is supported by NSF MCB 1818307. V.J.J.M. is supported by a Concordia University Research Chair.

\section{Author contributions}

M.E.P., K.K., P.S.G., L.N., J.E.D., and V.J.J.M. designed the research. M.E.P., K.K., P.S.G, and B.C. performed the experiments. L.N. assisted with HPLC and MS analyses and L.B. assisted in preliminary studies. V.J.J.M. and J.E.D. supervised the research. M.E.P., K.K., P.S.G., J.E.D., and V.J.J.M. wrote the manuscript with editing help from L.N., L.B., and B.C.

\section{Competing financial interests}

The authors declare competing financial interests.

\section{Additional information}

Supplementary information is available in the online version of the paper. Correspondence and requests for materials should be addressed to V.J.J.M. 


\section{ONLINE METHODS}

Plasmids, strains, and growth media. The quadruple auxotrophic S. cerevisiae strain BY4741

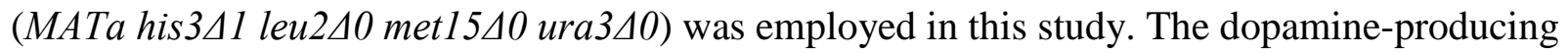
strain derived from BY4741 (strain 1373) was utilized as the basis for a yeast BIA platform strain. Yeast cultures were grown in YPD medium $\left(10 \mathrm{~g} \mathrm{~L}^{-1}\right.$ Bacto Yeast Extract, $20 \mathrm{~g} \mathrm{~L}^{-1}$ Bacto peptone, $20 \mathrm{~g} \mathrm{~L}^{-1}$ glucose). Transformed cells were selected on YPD agar containing $200 \mu \mathrm{g} \mathrm{mL}$ ${ }^{1}$ hygromycin $\mathrm{B}, 400 \mu \mathrm{g} \mathrm{mL} \mathrm{L}^{-1} \mathrm{G} 418$, or a combination of both antibiotics $\left(200 \mu \mathrm{g} \mathrm{mL} \mathrm{L}^{-1}\right.$ each). Selection using auxotrophic markers was performed in synthetic complete (SC) medium [6.7 $\mathrm{g} \mathrm{L}^{-}$ ${ }^{1}$ Difco Yeast Nitrogen Base (YNB) without amino acids, 1.62-1.92 $\mathrm{g} \mathrm{L}^{-1}$ Drop-out Medium Supplements (Millipore-Sigma) minus appropriate amino acids, $20 \mathrm{~g} \mathrm{~L}^{-1}$ glucose). Strains in which prototrophy was restored were selected on YNB medium $\left(6.7 \mathrm{~g} \mathrm{~L}^{-1}\right.$ Difco YNB, $20 \mathrm{~g} \mathrm{~L}^{-1}$ glucose). Plasmids and strains utilized in this work are listed in Supplementary Tables $\mathbf{3}$ and $\mathbf{4}$, respectively.

Yeast strain construction. All genetic modifications to yeast were made via CRISPR-Cas9mediated genomic integration ${ }^{50,51}$ and in vivo DNA assembly ${ }^{52}$. Cas9 and gRNA were delivered to yeast using pCas-G418 (ref. ${ }^{51}$ ) or a hygromycin-resistance derivative (pCas-Hyg) constructed herein. Linear gRNA cassettes were retargeted by PCR and assembled using in vivo gap repair with a linear PCR-generated pCas backbone ${ }^{50}$. Approximately $100 \mathrm{ng}$ of linear pCas was combined with $250 \mathrm{ng}$ of linear gRNA cassette and 500-1,000 ng of total repair DNA in a standard $50 \mu \mathrm{L}$ lithium acetate transformation. Cells were heat-shocked at $42{ }^{\circ} \mathrm{C}$ for 30 minutes, recovered for 16 hours, and plated onto YPD plates containing appropriate antibiotics. Both linear pCas-G418 and pCas-Hyg plasmids were used for transformations involving complex 
multi-part DNA assemblies. Design of gRNAs $^{53}$ and selection of chromosomal loci for DNA integration $^{30,54-56}$ were carried out according to previous studies. Gene expression cassettes, genomic integration sites, and synthetic DNAs utilized in this work are listed in Supplementary Tables 5-7, respectively.

Yeast genes targeted for deletion were replaced with a synthetic DNA landing pad (LP5.T3) possessing a unique Cas9 target site (T3) for subsequent genomic integration of additional copies of the $N d N C S \triangle N_{20}$ gene. Seven of the eight copies of $N d N C S \triangle N_{20}$ were later deleted in one genome editing event using the $N d N C S$ ( $\mathrm{T}_{P G I 1}$-LP5) gRNA and a single chromosomal LP5.T3 donor in the aad3 $\Delta$ locus. The remaining $N d N C S \Delta N_{20}$ copy was deleted from site FgF20 using an exogenous LP5.T3 donor. ALD4 was initially deleted using LP5.T3 and reintroduced in later strains along with its cognate promoter and terminator at a different $\operatorname{locus}(308 \mathrm{a})$.

Fluorescence microscopy. To visualize GFP-tagged proteins, cells from overnight cultures were back-diluted 50× into fresh SC medium and incubated at $30{ }^{\circ} \mathrm{C}$ and $200 \mathrm{rpm}$ for 4-6 hours. Cells were washed with water and mounted unfixed on microscope slides. Images were captured using a Nikon Ti microscope with a 100× PlanAPO lens (NA 1.49). Cells were illuminated using high inclination laminated optical sheet TIRF illumination with $488 \mathrm{~nm}$ lasers, and its respective filter cube (Chroma). Images are of single planes. Image processing was done using Fiji (NIH). Microtiter plate production assay for dopamine, BIAs, and fusel products. Colonies were picked in triplicate into $0.5 \mathrm{~mL}$ of $2 \times \mathrm{SCS}$ medium $\left(13.4 \mathrm{~g} \mathrm{~L}^{-1}\right.$ Difco Yeast Nitrogen Base (YNB) without amino acids, $2 \times$ Drop-out Medium Supplements (Millipore-Sigma) minus appropriate amino acids, $40 \mathrm{~g} \mathrm{~L}^{-1}$ sucrose) within 96-well deep well plates. Following 16-24 h of growth, saturated cultures were back-diluted $50 \times$ into $0.5 \mathrm{~mL}$ of fresh $2 \times$ SCS medium in 96-well deep 
well plates. Cultures were grown at $30{ }^{\circ} \mathrm{C}$ with shaking at $350 \mathrm{rpm}$. Following $72-96 \mathrm{~h}$ of growth, $\mathrm{OD}_{600}$ measurements were taken and culture broth was stored at $-20{ }^{\circ} \mathrm{C}$ for subsequent analysis by LC-MS.

\section{Cultivation of $(S)$-norcoclaurine and $(S)$-reticuline production strains in a pulsed fed-batch}

fermentor. Controlled fed-batch fermentations were carried out in $3 \mathrm{~L}$ BioBundle fermentors (Applikon). Cultivation temperature was maintained at $30^{\circ} \mathrm{C}$ and $\mathrm{pH}$ was kept at 4.5 by titration with $4 \mathrm{M} \mathrm{NaOH}$. Dissolved oxygen was maintained at $30 \%$ of air saturation by automatically adjusting the stirring rate (aeration rate $1.0 \mathrm{~L} \mathrm{~min}^{-1}$ ). Off-gas composition (concentration of $\mathrm{O}_{2}$ and $\mathrm{CO}_{2}$ ) was analyzed using a Tandem Multiplex gas analyzer (Magellan BioTech). Bioreactor inoculum was generated in two $250 \mathrm{~mL}$ shake flasks containing $50 \mathrm{~mL}$ of SC-His medium, grown for $24 \mathrm{~h}$ at $30{ }^{\circ} \mathrm{C}$. Cells were washed and suspended in $0.9 \% \mathrm{NaCl}$, and used to inoculate $\left(\mathrm{OD}_{600}=\sim 0.1\right) 1 \mathrm{~L}$ of batch medium (40 g sucrose, $2.5 \mathrm{~g} \mathrm{KH}_{2} \mathrm{PO}_{4}, 6.0 \mathrm{~g}\left(\mathrm{NH}_{4}\right)_{2} \mathrm{SO}_{4}, 1.0 \mathrm{~g}$ $\mathrm{MgSO}_{4} \cdot 7 \mathrm{H}_{2} \mathrm{O}, 1.92 \mathrm{~g} \mathrm{~L}^{-1}$ Drop-out Medium Supplements without histidine, $0.076 \mathrm{~g} \mathrm{~L}^{-1} \mathrm{~L}-$ tryptophan, $0.152 \mathrm{~g} \mathrm{~L}^{-1} \mathrm{~L}$-phenylalanine, $5 \mathrm{~mL}$ vitamin stock, and $5 \mathrm{~mL}$ trace element stock per liter). Vitamin and trace element stock solutions were based on a previous report ${ }^{57}$. The culture was operated in batch mode until sucrose was exhausted (24-30 h), followed by fed-batch phase with automated $10 \mathrm{~g} \mathrm{~L}^{-1}$ sucrose pulses (Supplementary Fig. 7). Feeding medium contained 360 g sucrose, $15 \mathrm{~g} \mathrm{KH}_{2} \mathrm{PO}_{4}, 60 \mathrm{~g}\left(\mathrm{NH}_{4}\right)_{2} \mathrm{SO}_{4}, 6 \mathrm{~g} \mathrm{MgSO} \cdot 7 \mathrm{H}_{2} \mathrm{O}, 4.16 \mathrm{~g}$ L-phenylalanine, $1.55 \mathrm{~g} \mathrm{~L}-$ tryptophan, $15 \mathrm{~mL}$ vitamin stock, and $15 \mathrm{~mL}$ trace element stock per liter. Samples were collected every 12 hours for a total of five days.

THIQ synthesis assay from supplemented amino acids. For growth on individual amino acids as the major source of nitrogen, reticuline-producing strain LP501 was transformed with pHUM (ref. ${ }^{58}$ ) to complement His and Met auxotrophies. Colonies were first picked in triplicate into 0.5 
$\mathrm{ml}$ of $2 \times \mathrm{YNB}$ (without amino acids and ammonium sulfate) containing $40 \mathrm{~g} \mathrm{~L}^{-1}$ sucrose and $1 \mathrm{~g}$ $\mathrm{L}^{-1}$ urea. Following $24 \mathrm{~h}$ of growth, saturated cultures were back-diluted $40 \times$ into $0.5 \mathrm{ml}$ of fresh 2× YNB (without amino acids and ammonium sulfate) containing $40 \mathrm{~g} \mathrm{~L}^{-1}$ sucrose within 96well deep well plates. Cultures of strain LP501 harboring pHUM were also supplemented with $0.076 \mathrm{~g} \mathrm{~L}^{-1}$ each of L-phenylalanine and L-tryptophan due to pha2 $\Delta$ and $\operatorname{trp} 3 \Delta$ mutations. For THIQ synthesis, cultures were supplemented with individual amino acids to a final concentration of $0.45 \mathrm{~g} \mathrm{~L}^{-1}$ (L-tyrosine), $2 \mathrm{~g} \mathrm{~L}^{-1}$ (L-2-aminoheptanoic acid, L-tryptophan, and L-DOPA), $3 \mathrm{~g} \mathrm{~L}^{-1}$ (L-norleucine), or $5 \mathrm{~g} \mathrm{~L}^{-1}$ (L-2-aminobutyrate, L-leucine, L-methionine, L-norvaline, and Lphenylalanine). Ascorbic acid (10 mM) was added to cultures supplemented with L-tryptophan, L-tyrosine, or L-DOPA. Cultures were grown at $30{ }^{\circ} \mathrm{C}$ with shaking at $350 \mathrm{rpm}$. Following $120 \mathrm{~h}$ of growth, $\mathrm{OD}_{600}$ measurements were taken and culture broth was stored at $-20{ }^{\circ} \mathrm{C}$ for subsequent analysis by LC-MS.

LC-MS and HPLC-UV analysis of metabolites. Dopamine, BIA, and other THIQ products from microtiter plate cultures were analyzed using HPLC-FT-MS. Metabolites were extracted from culture broth containing cells and growth medium. For early strains, $25 \mu \mathrm{L}$ of culture broth was combined with $100 \mu \mathrm{L}$ of cold $100 \%$ acetonitrile (ACN) and $542 \mu \mathrm{L}$ of $0.123 \%$ formic acid was added to give a final concentration of $15 \% \mathrm{ACN}$ and $0.1 \%$ formic acid. For more productive BIA strains and fed-batch cultures, an additional 10-fold dilution of samples was performed. Samples were centrifuged at 4,000 RCF and $10 \mu \mathrm{L}$ of extracted culture supernatant was separated on a 1290 Infinity II LC system (Agilent Technologies) with a Zorbax Rapid Resolution HT C18 column $(30 \times 2.1 \mathrm{~mm}, 1.8 \mu \mathrm{m}$; Agilent Technologies $)$. Metabolites were separated using the following gradient: $2 \% \mathrm{~B}$ to $10 \% \mathrm{~B}$ from $0-4 \mathrm{~min}\left(0.3 \mathrm{~mL} \mathrm{~min}^{-1}\right), 10 \% \mathrm{~B}$ to $85 \%$ B from $4-6 \min \left(0.3 \mathrm{~mL} \mathrm{~min}^{-1}\right)$, held at $85 \% \mathrm{~B}$ from $6-7 \mathrm{~min}\left(0.3 \mathrm{~mL} \mathrm{~min}^{-1}\right), 85 \%$ B to $2 \%$ 
B from 7-7.1 $\min \left(0.3 \mathrm{~mL} \mathrm{~min}^{-1}\right)$, and held at $2 \% \mathrm{~B}$ from $7.1-9 \mathrm{~min}\left(0.45 \mathrm{~mL} \mathrm{~min}^{-1}\right)$. Solvent A was $0.1 \%$ formic acid in water and solvent B was $0.1 \%$ formic acid in $100 \%$ ACN. Following separation, eluent was injected into an LTQ-FT-MS (Thermo Fisher Scientific) using 100 to 400 $\mathrm{m} / \mathrm{z}$ scanning range in positive mode. Resolution, capillary voltage, and source temperature were set to $100,000,5 \mathrm{kV}$, and $350^{\circ} \mathrm{C}$, respectively. FT-MS data was processed and manipulated using Xcalibur Qualitative Analysis software (Thermo Fisher Scientific).

Samples producing substituted THIQs from supplemented amino acids were analyzed using an Agilent 6545 quadrupole time-of-flight MS (QTOF-MS; Agilent Technologies) equipped with a Zorbax Eclipse Plus C18 column $(50 \times 2.1 \mathrm{~mm}, 1.8 \mu \mathrm{m}$; Agilent Technologies $)$ and using the aforementioned gradient conditions. The sample tray and column compartment were set to $4{ }^{\circ} \mathrm{C}$ and $30{ }^{\circ} \mathrm{C}$, respectively. The sheath gas flow rate and temperature were adjusted to $10 \mathrm{~L} \mathrm{~min}^{-1}$ and $350{ }^{\circ} \mathrm{C}$, respectively, while drying and nebulizing gases were set to $12 \mathrm{~L} \mathrm{~min}^{-1}$ and 55 psig, respectively. The drying gas temperature was set to $325^{\circ} \mathrm{C}$. QTOF data was processed and manipulated using Agilent MassHunter Qualitative Analysis software.

Dopamine and BIAs from fermentor samples and fusel products from both microtiter plate and fermentor cultures were analyzed and quantified using HPLC-UV according to a modified method ${ }^{44}$. Equal volumes of culture broth and $100 \%$ ACN containing $0.1 \%$ trifluoroacetic acid (TFA) were combined and samples were centrifuged at 4,000 RCF. Additional dilutions were performed as necessary. Five $\mu \mathrm{L}$ of extracted broth was separated on an Agilent 1200 HPLC system equipped with an Eclipse XDB-C18 column $(150 \times 4.6 \mathrm{~mm}, 5$ $\mu \mathrm{m}$, Agilent Technologies). Metabolites were separated using a flow rate of $1 \mathrm{~mL} \mathrm{~min} \mathrm{~m}^{-1}$ and the following gradient: $5 \% \mathrm{~B}$ to $20 \% \mathrm{~B}$ from $0-10 \mathrm{~min}, 20 \% \mathrm{~B}$ to $50 \% \mathrm{~B}$ from $10-15 \mathrm{~min}, 50 \% \mathrm{~B}$ to 95\% B from 15-15.1 min, and held at 95\% B from 15.1-25 min. Solvent A was 0.1\% TFA in 
water and solvent B was $0.1 \%$ TFA in $100 \%$ methanol. Tyrosol and 4-HPAC were detected at

$276 \mathrm{~nm}$; dopamine, $(S)$-norcoclaurine, and $(S)$-reticuline at $280 \mathrm{~nm}$.

\section{REFERENCES}

1 Cragg, G. M. \& Newman, D. J. Natural products: a continuing source of novel drug leads. Biochimica et Biophysica Acta (BBA)-General Subjects 1830, 3670-3695 (2013).

2 Veeresham, C. Natural products derived from plants as a source of drugs. Journal of Advanced Pharmaceutical Technology \& Research 3, 200-201 (2012).

3 Paddon, C. J. et al. High-level semi-synthetic production of the potent antimalarial artemisinin. Nature 496, 528-532 (2013).

4 Saxena, R., Anand, P., Saran, S. \& Isar, J. Microbial production of 1, 3-propanediol: recent developments and emerging opportunities. Biotechnol. Adv. 27, 895-913 (2009).

5 Meadows, A. L. et al. Rewriting yeast central carbon metabolism for industrial isoprenoid production. Nature 537, 694-697 (2016).

6 Diamond, A. \& Desgagné-Penix, I. Metabolic engineering for the production of plant isoquinoline alkaloids. Plant Biotechnol. J. 14, 1319-1328 (2016).

$7 \quad$ Khanna, K., Takido, M., Rosenberg, H. \& Paul, A. G. Biosynthesis of phenolic tetrahydroisoquinoline alkaloids of peyote. Phytochemistry 9, 1811-1815 (1970).

8 Lichman, B. R., Zhao, J., Hailes, H. C. \& Ward, J. M. Enzyme catalysed Pictet-Spengler formation of chiral 1, 1'-disubstituted-and spiro-tetrahydroisoquinolines. Nat. Commun. 8, 14883 (2017).

9 Ehrenworth, A. M. \& Peralta-Yahya, P. Accelerating the semisynthesis of alkaloid-based drugs through metabolic engineering. Nat. Chem. Biol. 13, 249 (2017).

10 Hagel, J. M. \& Facchini, P. J. Benzylisoquinoline alkaloid metabolism-a century of discovery and a brave new world. Plant Cell Physiol., 647-672 (2013).

11 Organization, W. H. The Selection and Use of Essential Medicines: Report of the WHO Expert Committee, 2015 (including the 19th WHO Model List of Essential Medicines and the 5th WHO Model List of Essential Medicines for Children). (World Health Organization, 2015).

12 Fossati, E. et al. Reconstitution of a 10-gene pathway for synthesis of the plant alkaloid dihydrosanguinarine in Saccharomyces cerevisiae. Nat. Commun. 5, 3283 (2014).

13 Fossati, E., Narcross, L., Ekins, A., Falgueyret, J.-P. \& Martin, V. J. Synthesis of morphinan alkaloids in Saccharomyces cerevisiae. PloS One 10, e0124459 (2015).

14 Hawkins, K. M. \& Smolke, C. D. Production of benzylisoquinoline alkaloids in Saccharomyces cerevisiae. Nat. Chem. Biol. 4, 564-573 (2008).

15 Li, Y. \& Smolke, C. D. Engineering biosynthesis of the anticancer alkaloid noscapine in yeast. Nat. Commun. 7, 12137 (2016).

16 DeLoache, W. C. et al. An enzyme-coupled biosensor enables $(S)$-reticuline production in yeast from glucose. Nat. Chem. Biol., 465-471 (2015).

17 Galanie, S., Thodey, K., Trenchard, I. J., Interrante, M. F. \& Smolke, C. D. Complete biosynthesis of opioids in yeast. Science 349, 1095-1100 (2015). 
18 Li, Y. et al. Complete biosynthesis of noscapine and halogenated alkaloids in yeast. PNAS, E3922-E3931 (2018).

19 Trenchard, I. J., Siddiqui, M. S., Thodey, K. \& Smolke, C. D. De novo production of the key branch point benzylisoquinoline alkaloid reticuline in yeast. Metab. Eng. 31, 74-83 (2015).

20 Matsumura, E. et al. Microbial production of novel sulphated alkaloids for drug discovery. Sci. Rep. 8, 7980 (2018).

21 Nakagawa, A. et al. Total biosynthesis of opiates by stepwise fermentation using engineered Escherichia coli. Nat. Commun. 7, 10390 (2016).

22 Smanski, M. J. et al. Synthetic biology to access and expand nature's chemical diversity. Nature Reviews Microbiology 14, 135-149 (2016).

23 Ignea, C. et al. Synthesis of 11-carbon terpenoids in yeast using protein and metabolic engineering. Nat. Chem. Biol. 14, 1090-1098 (2018).

24 Lichman, B. R. et al. 'Dopamine-first'mechanism enables the rational engineering of the norcoclaurine synthase aldehyde activity profile. The FEBS journal 282, 1137-1151 (2015).

25 Nishihachijo, M. et al. Asymmetric synthesis of tetrahydroisoquinolines by enzymatic Pictet-Spengler reaction. Biosci., Biotechnol., Biochem. 78, $701-707$ (2014).

26 Pesnot, T., Gershater, M. C., Ward, J. M. \& Hailes, H. C. The catalytic potential of Coptis japonica NCS2 revealed-Development and utilisation of a fluorescamine-based assay. Adv. Synth. Catal. 354, 2997-3008 (2012).

27 Ruff, B. M., Bräse, S. \& O’Connor, S. E. Biocatalytic production of tetrahydroisoquinolines. Tetrahedron Lett. 53, 1071-1074 (2012).

28 Hazelwood, L. A., Daran, J.-M., van Maris, A. J., Pronk, J. T. \& Dickinson, J. R. The Ehrlich pathway for fusel alcohol production: a century of research on Saccharomyces cerevisiae metabolism. Appl. Environ. Microbiol. 74, 2259-2266 (2008).

29 Wang, H. et al. Functions of aldehyde reductases from Saccharomyces cerevisiae in detoxification of aldehyde inhibitors and their biotechnological applications. Appl. Microbiol. Biotechnol. 102, 10439-10456 (2018).

30 Bourgeois, L., Pyne, M. E. \& Martin, V. J. A highly characterized synthetic landing pad system for precise multicopy gene integration in yeast. ACS Synth Biol. 7, 2675-2685 (2018).

31 Brockington, S. F. et al. Lineage-specific gene radiations underlie the evolution of novel betalain pigmentation in Caryophyllales. New Phytol. 207, 1170-1180 (2015).

32 Grewal, P. S., Modavi, C., Russ, Z. N., Harris, N. C. \& Dueber, J. E. Bioproduction of a betalain color palette in Saccharomyces cerevisiae. Metab. Eng. 45, 180-188 (2018).

33 Polturak, G. et al. Elucidation of the first committed step in betalain biosynthesis enables the heterologous engineering of betalain pigments in plants. New Phytol. 210, 269-283 (2016).

34 Sunnadeniya, R. et al. Tyrosine hydroxylation in betalain pigment biosynthesis is performed by cytochrome P450 enzymes in beets (Beta vulgaris). PLoS One 11, e0149417 (2016).

35 Payet, L.-A. et al. Mechanistic details of early steps in coenzyme Q biosynthesis pathway in yeast. Cell chemical biology 23, 1241-1250 (2016).

36 Zahedi, R. P. et al. Proteomic analysis of the yeast mitochondrial outer membrane reveals accumulation of a subclass of preproteins. Mol. Biol. Cell 17, 1436-1450 (2006). 
37 Beaudoin, G. A. \& Facchini, P. J. Benzylisoquinoline alkaloid biosynthesis in opium poppy. Planta 240, 19-32 (2014).

38 Allen, F., Pon, A., Wilson, M., Greiner, R. \& Wishart, D. CFM-ID: a web server for annotation, spectrum prediction and metabolite identification from tandem mass spectra. Nucleic Acids Res. 42, W94-W99 (2014).

39 Roddan, R. et al. The acceptance and kinetic resolution of alpha-methyl substituted aldehydes by norcoclaurine synthases. ACS Catalysis (2019).

40 O'Donovan, D. \& Horan, H. The biosynthesis of lophocerine. Journal of the Chemical Society C: Organic, 2791-2795 (1968).

41 Chen, X. et al. A pathogenesis-related 10 protein catalyzes the final step in thebaine biosynthesis. Nat. Chem. Biol 14, 738-743 (2018).

42 Dastmalchi, M. et al. Neopinone isomerase is involved in codeine and morphine biosynthesis in opium poppy. Nat. Chem. Biol., 384-390 (2019).

43 Kunjapur, A. M., Tarasova, Y. \& Prather, K. L. Synthesis and accumulation of aromatic aldehydes in an engineered strain of Escherichia coli. J. Am. Chem. Soc. 136, 1164411654 (2014).

44 Gold, N. D. et al. Metabolic engineering of a tyrosine-overproducing yeast platform using targeted metabolomics. Microb. Cell Fact. 14, 1 (2015).

45 Smolke, C. D., Thodey, C. \& Trenchard, I. (US Patent App. 15/567,354, 2018).

46 Atsumi, S., Hanai, T. \& Liao, J. C. Non-fermentative pathways for synthesis of branchedchain higher alcohols as biofuels. Nature 451, 86-89 (2008).

47 Billingsley, J. M. et al. Engineering the biocatalytic selectivity of iridoid production in Saccharomyces cerevisiae. Metab. Eng. 44, 117-125 (2017).

48 Kunjapur, A. M. \& Prather, K. L. Microbial engineering for aldehyde synthesis. Appl. Environ. Microbiol., AEM. 03319-03314 (2015).

49 Menéndez-Perdomo, I. \& Facchini, P. Benzylisoquinoline alkaloids biosynthesis in sacred lotus. Molecules 23, 2899 (2018).

50 Horwitz, A. A. et al. Efficient multiplexed integration of synergistic alleles and metabolic pathways in yeasts via CRISPR-Cas. Cell Systems, 88-96 (2015).

51 Ryan, O. W. et al. Selection of chromosomal DNA libraries using a multiplex CRISPR system. Elife 3, e03703 (2014).

52 Shao, Z., Zhao, H. \& Zhao, H. DNA assembler, an in vivo genetic method for rapid construction of biochemical pathways. Nucleic Acids Res. 37, e16-e16 (2009).

53 Labuhn, M. et al. Refined sgRNA efficacy prediction improves large-and small-scale CRISPR-Cas9 applications. Nucleic Acids Res. 46, 1375-1385 (2017).

54 Bai Flagfeldt, D., Siewers, V., Huang, L. \& Nielsen, J. Characterization of chromosomal integration sites for heterologous gene expression in Saccharomyces cerevisiae. Yeast $\mathbf{2 6}$, 545-551 (2009).

55 Mikkelsen, M. D. et al. Microbial production of indolylglucosinolate through engineering of a multi-gene pathway in a versatile yeast expression platform. Metab. Eng. 14, 104111 (2012).

56 Reider Apel, A. et al. A Cas9-based toolkit to program gene expression in Saccharomyces cerevisiae. Nucleic Acids Res. 45, 496-508 (2016).

57 Pyne, M. E. et al. An engineered Aro1 protein degradation approach for increased cis, cis-muconic acid biosynthesis in Saccharomyces cerevisiae. Appl. Environ. Microbiol. 84, e01095-01018 (2018). 
58 Mülleder, M., Campbell, K., Matsarskaia, O., Eckerstorfer, F. \& Ralser, M.

Saccharomyces cerevisiae single-copy plasmids for auxotrophy compensation, multiple marker selection, and for designing metabolically cooperating communities.

F1000Research 5 (2016). 